\title{
TRATAMENTO CIRÚRGICO DOS DIVERTÍCULOS EPIFRÊNICOS POR VIDEOLAPAROSCOPIA
}

\section{SURGICAL TREATMENT OF EPIPHRENIC DIVERTICULUM BY VIDEOLAPAROSCOPY}

\author{
Luiz Roberto Lopes, TCBC-SP'; ; Nelson Ary Brandalise, TCBC-SP²; \\ Nelson Adami Andreollo, TCBC-SP ${ }^{3}$
}

\begin{abstract}
RESUMO: Objetivo: O objetivo deste trabalho é registrar o tratamento cirúrgico de cinco pacientes operados por meio da videolaparoscopia, comentar a técnica empregada e apresentar os resultados obtidos. Método: São relatados cinco casos de tratamento cirúrgico videolaparoscópico de pacientes portadores de divertículo epifrênico. Três pacientes, cuja idade variou de 37 a 64 anos, eram do sexo feminino. Todos foram submetidos aos exames de endoscopia e contrastado do esôfago para confirmação do diagnóstico. A manometria foi realizada em três pacientes, que demonstrou alterações motoras inespecíficas. Resultados: Em todos os pacientes, a operação foi realizada por abordagem videolaparoscópica, tendo sido feita a ressecção do divertículo, hiatoplastia e fundoplicatura. A complicação mais importante foi uma fístula na linha de sutura do esôfago após oito dias, apesar de o exame contrastado de controle ter sido normal. O tempo de internação variou de 7 a 21 dias (média de 9,8 dias) e todos tiveram melhora da disfagia no acompanhamento de 2 a 11 anos. Conclusão: O tratamento cirúrgico do divertículo epifrênico pode ser realizado por videolaparoscopia, Essa via de acesso traz o benefício de um pós-operatório com menor dor, taxa de complicação aceitável e tempo de internação compatível com o porte da cirurgia. Por demonstrar ser segura e eficiente na resolução dos sintomas, hoje a técnica de escolha para o tratamento do divertículo epifrênico, em mãos experientes, pode ser considerada a primeira escolha (Rev. Col. Bras. Cir. 2006; 33(6): 365-368).
\end{abstract}

Descritores: Divertículo de esôfago; Cirurgia; Laparoscopia.

\section{INTRODUÇÃO}

A ocorrência dos divertículos epifrênicos na prática diária é rara ${ }^{1}$,e a maioria deles (62\%) são assintomáticos ou provocam poucos sintomas ${ }^{2}$.

Os divertículos localizam-se com mais freqüência nos $10 \mathrm{~cm}$ distais do esôfago, podendo ser acompanhados de alterações motoras muitas vezes difíceis de documentar, como acalásia, transtornos da motilidade do corpo do esôfago, espasmo difuso, hipertonia do esfíncter inferior e outras alterações não características, o que dificulta o reconhecimento da causa do problema ${ }^{3 ; 4}$.

Vinson $^{5}$ foi o primeiro a sugerir que, na gênese destes divertículos, poderia haver uma pressão intraluminal aumentada, causando o aparecimento dos divertículos em áreas de menor resistência. A acalásia foi a alteração manométrica mais encontrada. $\mathrm{Na}$ análise de 65 doentes portadores de divertículo epifrênico, observou-se que apenas 35\% deles apresentavam distúrbios motores ${ }^{6}$. Já Matthews et. al. ${ }^{7}$ demonstraram alterações motoras do esôfago à manometria, em três de cinco pacientes.

Os sintomas de disfagia, regurgitação, aspiração, tosse e complicações, como a infecção, a perfuração e a trans- formação neoplásica, são razões pelas quais o tratamento cirúrgico é recomendado ${ }^{3}$.

A maioria dos doentes com divertículos epifrênicos pequenos e com poucos sintomas não necessitam de tratamento, mas de acompanhamento e orientação ${ }^{8}$. Contudo, em raras ocasiões, são propostas dilatações do esfíncter inferior do esôfago?.

Assim, resta, para um grupo de doentes sintomáticos, a recomendação do tratamento cirúrgico. Como a operação é acompanhada por uma morbimortalidade não desprezível, a indicação depende muito dos sintomas que os pacientes apresentam ${ }^{10}$.

A operação era habitualmente realizada por toracotomia esquerda ou direita e consistia na ressecção do divertículo, sendo ou não associada à miotomia da cárdia e do esôfago distal ${ }^{11}$. Com os avanços da videocirurgia, esse procedimento passou a ser realizado e recomendado, tendo em vista os benefícios trazidos por essa abordagem cirúrgica ${ }^{12}$.

Os divertículos epifrênicos são uma ocorrência rara na prática clínica, sendo muitas vezes acompanhados de poucos sintomas, geralmente a disfagia. Antes do advento da videocirurgia, o tratamento era realizado por toracotomia esquerda ou por laparotomia e ressecção do divertículo associ-

1. Professor Doutor da Disciplina de Moléstias do Aparelho Digestivo do Departamento de Cirurgia da Faculdade de Ciências Médicas da Universidade Estadual de Campinas (UNICAMP) - Campinas - SP.

2. Professor Adjunto da Disciplina de Moléstias do Aparelho Digestivo do Departamento de Cirurgia da Faculdade de Ciências Médicas da Universidade Estadual de Campinas (UNICAMP) - Campinas - SP.

3. Professor Titular da Disciplina de Moléstias do Aparelho Digestivo do Departamento de Cirurgia da Faculdade de Ciências Médicas da Universidade Estadual de Campinas (UNICAMP) - Campinas - SP.

Recebido em 15/06/06

Aceito para publicação em 19/07/06

Conflito de interesses: nenhum

Fonte de Financiamento: nenhuma

Trabalho realizado na Disciplina de Moléstias do Aparelho Digestivo do Departamento de Cirurgia da Faculdade de Ciências Médicas da Universidade Estadual de Campinas (UNICAMP) - Campinas - SP. 
ada ou não à miotomia da transição esôfago-gástrica. Hoje, a cirurgia pode ser realizada por uma via minimamente invasiva que apresenta bons resultados, alcançando os benefícios do pós-operatório da videolaparoscopia.

\section{MÉTODO}

No período de 1994 a 2005, cinco pacientes com divertículo epifrênico foram diagnosticados, tratados e acompanhados na Disciplina de Moléstias do Aparelho Digestivo da Faculdade de Ciências Médicas da Unicamp. Três pacientes, cuja idade variou de 37 a 64 anos, eram do sexo feminino. A queixa principal era de disfagia há vários anos, sendo a maioria acima de cinco anos de história.

Todos os pacientes foram submetidos ao exame contrastado de esôfago-estômago-duodeno e à endoscopia digestiva alta, que confirmaram o diagnóstico de divertículo epifrênico, variando a sua localização em até $10 \mathrm{~cm}$ acima da transição esôfago gástrica. Três pacientes foram submetidos ao exame de manometria do esôfago que demonstrou distúrbios motores inespecíficos. Uma paciente apresentava hérnia hiatal pequena sem esofagite de refluxo e outro havia sido submetido à correção de refluxo há oito anos em outro serviço, pela técnica de Lind.

O tratamento indicado para os três pacientes foi a ressecção do divertículo, fechamento do hiato diafragmático e a realização de uma fundoplicatura parcial à maneira de Lind ${ }^{13}$. Para os outros dois, além da ressecção do divertículo e do fechamento do hiato, foi também indicada uma fundoplicatura de Nissen Modificada ${ }^{14}$ (técnica mista).

A operação videolaparoscópica indicada foi aquela habitualmente empregada para a abordagem da transição esôfago-gástrica na correção do refluxo gastroesofagiano. Após a dissecção do divertículo, este foi ressecado por meio do uso do endogrampeador linear 60. A seguir, o hiato foi aproximado e a fundoplicatura foi realizada à maneira de Lind ${ }^{13}$ ou pela técnica de Nissen modificada ${ }^{14}$.

Os cuidados pós-operatórios empregados foram concernentes ao início da alimentação. Os pacientes foram mantidos em jejum por via oral por um período de 5 a 7 dias, quando, então foi realizado o estudo contrastado do esôfago para confirmação do trânsito normal e da ausência de fístula. A nutrição, nesse período, foi feita por sonda naso-enteral deixada ao final do ato operatório.

\section{RESULTADOS}

A primeira paciente operada apresentou um pneumotórax à direita, que foi drenado. Uma paciente apresentou fístula no décimo dia de pós-operatório, apesar do exame contrastado ter sido normal antes do início da alimentação, permanecendo internada até o fechamento da fístula, por 21 dias, em tratamento com alimentação parenteral total. Outro paciente apresentou empiema pleural à esquerda, tratado por drenagem torácica. Nesse mesmo paciente foi descoberto, no intra-operatório, um leiomioma da parede esofagiana, que foi ressecado, e o exame anátomo-patológico confirmou o diagnóstico. O tempo de internação variou de 7 a 21 dias, com média de 9,8 dias de internação. O estudo anátomo-patológico dos divertículos ressecados não mostrou particularidades. Todos os pacientes foram submetidos ao estudo radiológico contrastado e ao exame endoscópico no pós-operatório, mostrando pequenos recessos no esôfago distal. O acompanhamento desses pacientes variou de 2 a 11 anos, com melhora significativa dos sintomas.

\section{DISCUSSÃO}

O diagnóstico dos divertículos epifrênicos é feito baseado na história clínica e confirmado pelo exame radiográfico contrastado do esôfago. O exame de manometria do esôfago tem sua indicação para o estudo das alterações motoras, pois estas podem estar associadas e seu conhecimento é fundamental para orientação segura da terapêutica. As alterações manométricas podem ser encontradas em cerca de $35 \%$ dos pacientes ${ }^{6}$. Em nosso estudo, o exame manométrico não foi realizado em todos os pacientes por dificuldade na passagem do cateter pelo divertículo, ou por não apresentarem alterações motoras importantes ao exame contrastado.

A endoscopia digestiva alta é indicada para completar o estudo da mucosa do divertículo e do esôfago, afastando outros diagnósticos.

Os pacientes sintomáticos e os que apresentam grandes divertículos merecem tratamento cirúrgico. O tratamento conservador expectante pode ser recomendado em pacientes com risco para cirurgia ou com pequenos divertículos e assintomáticos ${ }^{4}$.

Quando da indicação de tratamento cirúrgico, o acesso é preferencialmente realizado por toracotomia, em que se faz a ressecção do divertículo, seguido de miotomia da cárdia e do esôfago distal, havendo indicação. Existe a recomendação de se realizar gastrofundoplicatura. Essa via de abordagem é acompanhada de morbidade, representada por dor e hemopneumotórax, razão pela qual o tratamento é muitas vezes postergado ${ }^{11}$.

Nos últimos anos, com o advento da videotoracoscopia e da videolaparoscopia, a resseção dos divertículos passou a ser realizada por uma dessas vias, com bons resultados ${ }^{15,16}$.

A abordagem pela via laparoscópica tem a vantagem de também permitir a realização de outros procedimentos na cavidade abdominal.

A operação de escolha tem sido a ressecção do divertículo com auxílio de grampeador endoscópico, que tanto pode ser por toracoscopia, ${ }^{10,3}$ como por laparoscopia ${ }^{4,7,17-18}$ embora, em casos isolados, a elevação do divertículo tenha sido citada $^{3,4}$.

O exame endoscópico intra-operatório foi utilizado por Renz et. $a l .{ }^{19}$ para assegurar a completa ressecção do divertículo, método que consideramos desnecessário. Utilizamos uma sonda nasogástrica $n^{\circ} 20$ para auxiliar na identificação do divertículo e, no momento da sua ressecção evitar a estenose do esôfago.

Dos cinco pacientes operados e aqui apresentados, três foram submetidos a estudo manométrico do esôfago no pré-operatório e não mostraram alterações motoras importan- 
tes, de modo que foi realizada a ressecção do divertículo. Os outros dois pacientes, mesmo sem o estudo manométrico, foram submetidos também à ressecção do divertículo. Jordan \& Kinner ${ }^{8}$ operaram dezenove pacientes e não realizaram a miotomia em oito deles. Desses oito, quatro tiveram excelente resultado. Matthews et. al. ${ }^{7}$ observaram alterações mínimas motoras em três de cinco pacientes que foram submetidos a estudo manométrico no pré-operatório, mas ainda assim realizaram miotomia e fundoplicatura em todos, com alívio dos sintomas.

A gastrofundoplicatura foi realizada em todos os doentes, pois a ampla dissecção da região da transição esôfago-gástrica exigiu tal procedimento, para evitar o refluxo gastroesofagiano.

As complicações encontradas foram uma fistula na linha de sutura no décimo dia de pós-operatório. Essa complicação não foi observada no exame radiográfico realizado no oitavo dia de pós-operatório. O tratamento consistiu de drenagem de tórax e nutrição parenteral total, tendo boa evolução. Na literatura, encontramos uma casuística de dezenove pacientes operados por Jordan \& Kinner, ${ }^{8}$ em que foi observado uma fístula, tratada clinicamente com fechamento espontâneo. van der Peet et. al. ${ }^{10}$ operaram cinco pacientes e descreveram uma fístula que foi tratada por toracotomia. Klaus e.t $a l .{ }^{4}$ fizeram tratamento cirúrgico em onze pacientes e tiveram uma fístula pós-operatória. A ocorrência da fístula não é rara, mas apresenta evolução satisfatória nas diversas casuísticas descritas.

Outra complicação encontrada foi empiema pleural tratado com drenagem aberta de tórax. Provavelmente o empiema foi em decorrência de derrame pleural pós-operatório infectado. Pelo fato de se abordar o mediastino e de se realizar uma ampla dissecção da área, é possível ocorrerem lesões de pleura não identificadas no intra-operatório. Klaus et. al. $^{4}$ também relataram um caso de empiema quatro semanas após a cirurgia.

De maneira geral, as complicações pós-operatórias existem, sendo muitas relacionadas à cavidade pleural e de resolução satisfatória, não havendo mortalidade referida nos diversos trabalhos consultados na literatu$\mathrm{ra}^{3,4,7,10,17,18}$. Vale a pena lembrar que o tratamento cirúrgico pela toracotomia ou toracoscopia implicaria o uso de drenos, obrigatoriamente, em todos os pacientes. Seu uso na laparoscopia é necessário em casos de complicações como as aqui descritas.

Os cinco pacientes tratados nessa casuística ficaram satisfeitos com o resultado da cirurgia, apresentando sintomas disfágicos esporádicos no pós-operatório com acompanhamento de dois a onze anos.

Diante de um paciente com diagnóstico de divertículo epifrênico e com poucos sintomas, há dúvidas quanto à indicação do tratamento cirúrgico. O dilema ocorre, em especial, se existirem alterações motoras que possam ser responsáveis pelos sintomas, ou divertículos de grande tamanho. Porém, diante da decisão por um tratamento cirúrgico, deve-se optar pela técnica que forneça segurança e baixa morbidade. $\mathrm{O}$ tratamento cirúrgico do divertículo epifrênico por videolaparoscopia é possível ser realizado. Essa via de acesso traz o benefício de um pós-operatório com menor dor, taxa de complicação aceitável e tempo de internação compatível com o porte da cirurgia. Por demonstrar ser segura e eficiente na resolução dos sintomas, hoje a técnica videolaparoscópica para o tratamento do divertículo epifrênico, em mãos experientes, pode ser considerada a primeira escolha.

\begin{abstract}
Background: Epiphrenic diverticulum is a rare condition in clinical practice usually presented by few symptoms such as dysphagia. Before the advent of the videosurgery, it's surgical treatment was performed by a left thoracotomy or laparotomy and resection of the diverticulum, associated or not with myotomy in the esophagogastric junction. Today, this procedure has been accomplished, by laparoscopy with very good results, leading to a better postoperative recuperation. Methods: We present five patients with epiphrenic diverticulum that were treated by videolaparoscopy. Three of them were females and their ages varied between 37 and 64 years old. All patients were submitted to an endoscopy and X-ray to confirm the diagnosis; three were submitted to a manometry that showed unspecific motility alterations. Results: Resection of the diverticulum was performed successfully by videolaparoscopy in all cases. One patient showed esophageal leakage after 8 days with normal control postoperative X-ray. The hospital length of stay varied between 7 and 21 days, and disphagya relief was achieved in all patients, with a follow-up between two to eleven years. Conclusion: Videolaparoscopic surgical repair for epiphrenic diverticulum, associated or not to myotomy of the esophagogastric junction, is the treatment of choice. It is associated with less pain and complications, with a shorter length of stay. This technique is safe and efficient for treatment of the epiphrenic diverticulum.
\end{abstract}

Key words: Esophageal diverticulum; Surgery; Laparoscopy.

\section{REFERÊNCIAS}

1. Allen MS. Treatment of epiphrenic diverticula. Semin Thorac Cardiovasc Surg. 1999 Oct;11(4):358-62.

2. Benacci JC, Deschamps J, Trostek VF, Allen MS, Daly RC, Pairolero P. Epiphrenic diverticulum: results of surgical treatment. Ann Thorac Surg. 1993 May;55(5):1109-13; discussion 1114. Comment in: Ann Thorac Surg. 1993 May;55(5):1067-8.
3. Nehra D, Lord RV, DeMeester TR, Theisen J, Peters JH, Crookes $\mathrm{PF}$, Bremner CG. Physiologic basis for the treatment of epiphrenic diverticulum. Ann Surg. 2002 Mar;235(3):346-54.

4. Klaus A, Hinder RA, Swain J, Achem SR. Management of epiphrenic diverticula. J Gastrointest Surg. 2003 Nov;7(7):906-11.

5. Vinson PP. Diverticulla of the thoracic portion of the esophagus. Report of 42 cases. Arch. Otolaryngol. 1934;19:508-13. 
6. Debas HT, Payne WS, Cameron AJ, Carlson HC. Physiophatology of lower esophageal divertuculum and its implications for treatment. Surg Gynecol Obstet. 1980 Nov;151(5):593-600.

7. Matthews BD, Nelms CD, Lohr CE, Harold KL, Kercher KW, Heniford BT. Minimally invasive management of epiphrenic esophageal diverticula. Am Surg. 2003 Jun;69(6):465-70; discussion 470.

8. Jordan PH, Kinner BM. New look at epiphrenic diverticula. World J Surg. 1999 Feb;23(2):147-52.

9. Orringer M.B. Epiphrenic diverticula. Fact and fable. Ann Thorac Surg. 1993 May;55(5):1067-8.

10. van der Peet DL, Klinkenberg-Knol EC, Berends FJ, Cuesta MA. Epiphernic diverticula: minimal invasive approach and repair in five patients. Dis. Esophagus. 2001;14(1):60-2.

11. Chami Z, Fabre JM, Navarro F, Domergue J. Abdominal laparoscopic approach for the thoracic epiphrenic diverticulum. Surg Endosc. 1999 Feb;13(2):164-5.

12. Rosati R, Fumagalli U, Bona S, Bonavina L, Peracchia A. Diverticulectomy, myotomy, and fundoplication through laparoscopy. A new option to treat epiphrenic esophageal diverticula? Ann Surg. 1998 Feb;227(2):174-8.

13. Lind JF, Burns CM, MacDougall C. "Physiological" repair for hiatus hernia - Manometric study. Arch. Surg. 1965; 91:233-237.

14. Lopes LR, Brandalise NA, Andreollo, NA, Leonardi, LS. Tratamento cirúrgico videolaparoscópico da doença do refluxo gastroesofagiano: técnica de Nissen modificada - resultados clínicos e funcionais. Rev. Assoc. Med. Bras., 2001; 47(2):141-148.

15. Saw EC, McDonald TP, Kam NT. Video-assisted thoracoscopic resection of an epiphrenic diverticulum with esophagomyotomy and partial fundoplication. Surg Laparosc Endosc. 1998 Apr;8(2):145-8.
16. Chiappalone S, Russo B, Masella C, Fontana B, Virgilio D. The epiphrenic esophageal diverticulum. Transhiatal laparoscopic treatment. Minerva Chir. 1999 Sep;54(9):631-4.

17. Fraiji EJr, Bloomston M, Carey L, Zervos E, Goldin S, Banasiak M, Wallace M, Rosemurgy AS. Laparoscopic management of symptomatic achalasia associated with epiphrenic diverticulum. Surg Endosc. 2003 Oct;17(10):16003. Epub 2003 Jul 21.

18. Del Genio A, Rosseti G, Maffetton V, Renzi A, Brusciano L, Limongelli P, Cuttita D, Russo G, Del Genio G. Laparoscopic approach in the treatment of epiphrenic diverticula: long-term results. Surg Endosc. 2004 May;18(5):741-5.

19. Renz EM, Parker MV, Hetz SP. Laparoscopic repair of a large symptomatic epiphrenic esophageal diverticulum. Curr Surg. 2002 Mar-Apr;59(2):190-3

Como citar este artigo:

Lopes LR, Brandalise NA, Andreollo NA. Tratamento cirúrgico dos divertículos epifrênicos por vídeolaparoscopia. Rev Col Bras Cir. [periódico na Internet] 2006 Nov-Dez;33(6). Disponível em URL: http://www.scielo.br/rcbc

Endereço para correspondência:

Luiz Roberto Lopes

Rua Amélia Maria de Paula Venturini 365

Parque das Universidades

13086-061 - Campinas - SP

Fone: 0(xx)19-3256-5990

E-mail: lopes@unicamp.br 